Meta

Journal des traducteurs

Translators' Journal

\title{
Téléologie de la traduction universitaire
}

\section{Michel Ballard}

Volume 50, numéro 1, mars 2005

Enseignement de la traduction dans le monde

Teaching Translation Throughout the World

URI : https://id.erudit.org/iderudit/010656ar

DOI : https://doi.org/10.7202/010656ar

Aller au sommaire du numéro

Éditeur(s)

Les Presses de l'Université de Montréal

ISSN

0026-0452 (imprimé)

1492-1421 (numérique)

Découvrir la revue

\section{Citer cet article}

Ballard, M. (2005). Téléologie de la traduction universitaire. Meta, 50(1), 48-59. https://doi.org/10.7202/010656ar

\section{Résumé de l'article}

Le drame de la traduction à l'université, c'est qu'elle n'est généralement pas accompagnée d'un cours de traductologie qui finalise cette activité et lui donne les moyens d'y parvenir. On propose ici un cadre pour les mettre en évidence autour de deux axes principaux : la prise en compte de l'apprenant et une découverte ordonnée de la nature de la traduction qui allie l'exposé de perspectives théoriques larges à l'observation scientifique de la réalité.
Ce document est protégé par la loi sur le droit d'auteur. L'utilisation des services d’Érudit (y compris la reproduction) est assujettie à sa politique d'utilisation que vous pouvez consulter en ligne.

https://apropos.erudit.org/fr/usagers/politique-dutilisation/ 


\title{
Téléologie de la traduction universitaire
}

\author{
MICHEL BALLARD \\ Université d'Artois, Artois, France \\ michel.ballard@univ-artois.fr
}

\begin{abstract}
RÉSUMÉ
Le drame de la traduction à l'université, c'est qu'elle n'est généralement pas accompagnée d'un cours de traductologie qui finalise cette activité et lui donne les moyens d'y parvenir. On propose ici un cadre pour les mettre en évidence autour de deux axes principaux: la prise en compte de l'apprenant et une découverte ordonnée de la nature de la traduction qui allie l'exposé de perspectives théoriques larges à l'observation scientifique de la réalité.
\end{abstract}

\begin{abstract}
The pity with translation as an Academic subject is that it is rarely sustained by a regular course in translation studies clearly stating the aims of this activity and providing the means to achieve them. This article proposes to bring them out along two main lines: the taking into account of the needs and failings of learners on one hand and on the other a gradual and ordered discovery of the nature of translation through a general presentation of theoretical problems tempered and inevitably bound and matched with a regular and scientific observation of the reality of translation.
\end{abstract}

\section{MOTS-CLÉS/KEYWORDS}

nature de la compétence, enseignement du lexique, analyse d'erreurs, observation de la traduction, lecture et construction du sens

\section{En guise d'introduction : un bref état des lieux}

La traduction figure en bonne place dans les cursus universitaires français alors qu'elle est beaucoup moins présente dans les cursus des universités anglaises ou américaines. Dans de nombreux pays, tout comme en France (surtout au niveau de l'enseignement secondaire), on estime même que cette activité n'est pas nécessaire à l'apprentissage des langues et une grande partie des attaques qui ont été adressées à la traduction ont joué sur cet argument: elle n'est pas un moyen d'expression naturel et bloquerait l'expression en langue étrangère. Dans certains pays, la traduction tendrait même à être évacuée de l'enseignement supérieur proprement dit pour se cantonner dans les formations professionnalisantes. Quand elle demeure, c'est souvent sous des formes assez marginales, et cette marginalisation reflète, dans une certaine mesure, le statut de la traduction dans la société (même si celui-ci évolue de façon positive) ainsi que le statut encore incertain de la traductologie dans l'institution universitaire. La traduction demeure donc mais avec des finalités et des modalités qui sont sinon floues tout au moins variables.

Un certain nombre d'universités possèdent aujourd'hui un enseignement de traductologie, mais c'est plutôt rare (je suis frappé, lorsque je commence un cours de maîtrise ou de DEA, par le faible nombre d'étudiants qui ont suivi des cours de traductologie ou qui ont lu des ouvrages de traductologie). Je considère que l'état des 
lieux que je vais continuer d'esquisser dans le paragraphe suivant part d'un état d'esprit et d'un état de fait moyen, encore assez répandu (trop, hélas! à mon sens).

Pour certains enseignants, la traduction est (même si c'est de façon inavouée) un moyen assez économique de faire cours, qui ne demande pas un trop grand investissement (tout le monde sait traduire, dès qu'il connaît une langue étrangère, du moins c'est ce que certains estiment), elle est aussi, de l'avis presque général, un moyen assez pratique, et efficace, de tester des connaissances linguistiques. Pour les étudiants, la traduction est un moyen d'avoir un examen ou un concours en montrant, ou en prouvant, par leur capacité à traduire, la possession d'une certaine intelligence et un certain degré de connaissances; elle est aussi, de leur point de vue, un cours moins astreignant que les autres, puisqu'il n'y a aucun programme, aucune somme de connaissance, à digérer; on vient au cours avec son poly, en ayant préparé (mais aussi sans avoir préparé) un texte auquel on daignera collaborer selon l'humeur ou l'inspiration. Le cours de traduction est essentiellement un cours de pratique où l'on fait de la traduction de semaine en semaine en espérant parvenir à un certain degré de compétence par une pratique intuitive assaisonnée de remarques faites au fil des textes et dont l'objet est purement ponctuel puisqu'il ne vise que l'effectuation du texte en cours (au lieu d'être rapporté à une vision d'ensemble).

Une telle situation ne peut manquer de susciter quelques questions quant aux finalités et aux modalités de cet "enseignement»; faut-il entériner un état de fait fondé sur la routine et la facilité, ou prendre de la hauteur pour essayer de repenser les objectifs et les procédures d'un enseignement qui apparaît beaucoup moins noble que la triade institutionnelle: linguistique, civilisation et littérature.

\section{Quelques premières interrogations et propositions :}

Une question fondamentale, à laquelle, on le verra, je vais revenir et répondre par des voies détournées, serait de se demander si l'objectif essentiel de la traduction à l'université est un test et de servir à avoir des examens ou des concours, alors que le cours de littérature vise à faire connaître la chose littéraire, à réfléchir sur l'objet littérature; le cours de civilisation enseigne ce qu'est la civilisation; le cours de linguistique enseigne des théories du langage et aide, à réfléchir sur le langage. Mais pour le moment, soyons docile et conventionnel, et acceptons ce but, cet objectif; on est endroit, quand même, à partir de là, de se poser quelques questions.

\subsection{Quelle doit être la nature de la compétence exigée de l'étudiant?}

Si l'on conserve comme objectif pour la traduction d'être une préparation à une compétence, on peut se demander tout d'abord quelle doit être la nature de cette compétence: s'agit-il de traduire comme les professionnels ou de traduire selon une manière que certains qualifient d'artificielle ou d'académique, ou d'universitaire? Faut-il continuer de faire cours en s'excusant ou en répétant aux étudiants (dès que l'on est prêt à accepter certaines formulations qui peuvent paraître inusitées ou audacieuses): «Ce que vous dites est possible, mais je ne le mettrais pas dans une copie de CAPES ou d'agrégation. Faut-il que la traduction à l'université soit décalée par rapport au monde réel?» 
Je ne prendrai pas ici parti de façon tranchée car, à mon sens, le problème est complexe et touche en réalité à celui des manières de traduire; il y a derrière ce problème celui des critères de traduction : vaste problème, mais qu'il ne faudrait pas éluder et dont la présence est perceptible derrière certaines des inquiétudes réelles et justifiées des étudiants, qui parfois se demandent à quelle sauce ils vont être mangés. Le débat est vaste, car il faudrait à la fois faire la part des manières de traduire des professionnels (elles sont loin d'être homogènes) et dédouaner aussi la ou plutôt les manières de traduire des universitaires. Il existe des théoriciens issus des rangs des professionnels qui ont tendance à rejeter la traduction universitaire en bloc, alors qu'il y a des traducteurs universitaires capables non seulement d'exigence mais aussi d'élégance.

\subsection{Comment améliorer cette compétence?}

La seconde question qu'il convient de se poser est de savoir comment améliorer cette compétence, ce qui suppose une interrogation sur ses fondements. Il me semble que la compétence traductive repose sur deux types de facteurs au moins: des facteurs qui sont de l'ordre de la connaissance et d'autres qui sont de l'ordre du savoir-faire. Je parlerai d'abord des premiers.

Les écoles de traducteurs, ou les formations professionnalisantes (de type DESS), exigent de leurs candidats une très haute connaissance des deux langues impliquées dans la traduction, certains parlent même de bilinguisme. C'est loin d'être le cas des étudiants et en particulier des étudiants de Deug (de première et seconde année) et même au-delà; en ce sens, la traduction à l'université, tout au moins dans les premières années est presque un non-sens: c'est une machine à faire des fautes, une machine à échec ou alors à sélection. Quelle en est la cause?

Une machine pour fonctionner a besoin d'être alimentée. L'alimentation linguistique de l'étudiant est en partie assurée, et de façon fort compétente, par nos collègues linguistes ou grammairiens; mais, comme nous le savons, une langue n'est pas faite que de structures et d'opérations (fussent-elles énonciatives!) : le lexique est une composante majeure du langage et une part très importante des problèmes de traduction est liée au lexique. Or, face à ce champ immense, et alors que l'on s'est plu depuis Saussure à souligner le fait que le lexique n'était pas un simple répertoire, une simple liste de mots, c'est souvent encore la méthode qui domine pour son apprentissage, puisque l'on finit toujours par renvoyer l'étudiant aux fameuses listes de vocabulaire qu'il doit pratiquer seul, avec les résultats que l'on sait (certains ne vont même pas jusque-là, et se contentent du vocabulaire glané au fil des traductions), la seule nuance introduite étant qu'il faut beaucoup lire et parler pour affiner sa connaissance du lexique. Ceci est vrai, mais, comme on le voit, ce à quoi on nous renvoie est une acquisition intuitive, dont nous savons qu'elle ne se fera pas, dans la mesure où les étudiants lisent peu et vont peu à l'étranger.

De même qu'il y a un enseignement de linguistique et un enseignement structuré de grammaire, il serait bon d'introduire un enseignement structuré du lexique avec des cours et des exercices en amont ou en parallèle du cours de traduction. Le cours de traduction prendrait alors un peu moins cette allure de jeu télévisé où une assemblée lance au hasard en pâture à l'enseignant des fragments de lexique en espérant que, par miracle, il y en aura un qui finira par s'insérer dans le puzzle que l'on est en train de construire. 
Je parlerai maintenant du second facteur sur lequel repose la compétence traductive: le savoir-faire. On peut se demander si faire de la traduction (et en particulier une trentaine de traductions) est la seule et meilleure manière de s'améliorer dans le domaine. Les professionnels eux-mêmes reconnaissent qu'il est nécessaire d'avoir une formation théorique à côté de la formation pratique (voir entre autres, Delisle, 1980; Durieux, 1988; Balliu, 1999) et je me permettrai d'ajouter que dans un cadre universitaire il est souhaitable que cette formation soit réflexive et ce sur au moins deux niveaux ou dans deux sens.

Tout d'abord, il me semble important de promouvoir une réflexivité sur l'apprenant; nous vivons à une époque où tout individu ayant une activité, et cherchant à l'améliorer, pratique une forme d'observation de soi: les sportifs enregistrent leurs performances, les artistes font de même; il est désolant de constater que le cours de traduction prend souvent l'allure d'une course orientée vers la production d'un texte adéquat, généralement celui de l'enseignant au lieu, surtout dans les premiers stades, de se tourner vers les besoins de l'apprenant qui transparaissent au travers de ses fautes. Le second niveau auquel je placerais la réflexivité c'est celui de l'opération elle-même, et il conviendra de mettre en relation, de faire communiquer, les deux niveaux, précisément par le biais de la réflexion: en posant l'incompétence de l'étudiant comme un déficit mesurable par rapport à une compétence idéale ou réelle objectivée.

\subsection{La prise en compte de l'apprenant: l'erreur comme base de travail}

Se pose ici le problème de la composition du public, de son niveau et de son éventuelle et même probable hétérogénéité. On ne traitera pas de la même façon un public de futurs traducteurs et un public d'étudiants dans le cadre d'une formation universitaire ou de la préparation à un concours; et les besoins ne seront pas les mêmes pour un public étudiant que pour celui d'une préparatoire.

La démocratisation de l'enseignement supérieur en France a amené à l'université des étudiants de niveaux très différents et aux objectifs non moins divers: certains veulent effectuer des études classiques, certains visent ou aimeraient une certaine professionnalisation de leurs études, d'autres enfin sont en attente d'autre chose: ils se cherchent. Le facteur le plus commun par lequel l'enseignant peut les aborder est la prise en compte de leurs problèmes face à la traduction, même si ceux-ci sont spécifiques, et de les traiter par rapport à leurs propres productions, ce qui implique forcément la prise en compte de l'erreur.

L'erreur est souvent traitée de façon censoriale ou traumatisante, les deux comportements étant étroitement liés, et d'ailleurs étroitement liés à la finalité implicite ou explicite de la traduction dans l'enseignement. La traduction figure aux examens et aux concours comme test de langue, et il s'agit d'avoir une bonne note pour réussir son examen: il s'agit donc de montrer que l'on est capable de faire une bonne traduction et donc il convient d'éviter l'erreur et de lui substituer une traduction correcte. L'erreur s'accompagne de toute façon d'un sentiment désagréable d'infériorité ou de ridicule qui fait que l'on préfere ne pas s'y attarder; et, de plus, sur le plan de la conduite du cours, il est plus rapide de lui substituer une performance correcte comme terme définitif qui clôt de toute façon le dialogue, si tant est qu'il ait eu lieu. J'ai toujours noté, lorsque je commence à faire cours avec un groupe, que si un étudiant 
me propose une mauvaise traduction et que je demande aux autres de la commenter, le plus souvent ils me donnent leur traduction en espérant qu'elle sera la bonne; je le répète: l'objectif implicite derrière ces comportements est un objectif de productivité, de conformation à un modèle et de passage dans un cercle de compétence reconnue. Ce système a longtemps fonctionné de façon satisfaisante pour certains : il est évident que l'accession de couches plus larges de populations estudiantines à ce système les laisse surtout sur un sentiment de rejet et d'insatisfaction assez traumatisant. Le problème qui est également lié à ces comportements est de savoir si la compétence est innée, auquel cas l'enseignement ne sert à rien; ou encore de savoir si elle s'acquièrent par la simple performance, auquel cas le passage à l'Université est inutile ou devrait être transformé en stage de formation professionnel; ou bien alors cette fameuse compétence peut-elle être éclairée par la réflexion, et comment? Si l'on s'oriente vers la troisième option, la traduction peut alors remplir une fonction typiquement universitaire, qui est la réflexion sur un objet, et, dans ce cas, l'objet est multiple: la traduction, son exécution, son estimation, son analyse et l'utilisation de son analyse à des fins de performance. Tels sont à mon avis les finalités que l'on peut proposer à la traduction en milieu universitaire, pour le bien de cette activité elle-même, pour celui de l'institution et celui de l'individu qui vient y chercher une formation de l'esprit tout autant qu'un affinement ou un renforcement de ses compétences linguistiques et autres.

Le premier objectif que je fixerais donc à une didactique de la traduction serait de créer la capacité à discourir sur la traduction, à commenter les performances satisfaisantes ou non; en un mot, à être capable de porter un jugement justifié sur la traduction et d'abord à partir de l'erreur. Ceci suppose la mise en place d'un métalangage spécifique, qui permette de tenir un discours sur la traduction lié à la nature de celle-ci, c'est-à-dire un discours d'ordre traductologique.

\section{Programme: une découverte de la nature de l'opération traduisante, construite autour de ses axes fondamentaux}

La didactique de la traduction doit reposer sur une prise en compte des acteurs et de l'acte, c'est-à-dire sur une prise en compte de leurs problèmes et d'une réflexion générale sur l'acte. En d'autres termes, la didactique de la traduction doit reposer sur une théorie de la traduction: elle doit partir des problèmes des apprenants pour amener ces derniers à une perception générale et plus fine des problèmes de traduction. Au cour de la traduction, il y a un phénomène complexe dont nous essayons, pour des raisons de réflexion et de transmission du savoir, de dissocier les composantes alors que tout se passe très vite. On dit souvent qu'il faut soigneusement lire le texte avant de le traduire, mais souvent, dès qu'on lit on traduit et dès qu'on traduit on estime sa production, on juge. Ce sont les trois composantes liées autour desquelles j'organiserais une didactique et de manière générale une théorisation de la traduction: lecture, reformulation et jugement concernant l'équivalence et la lisibilité. J'en exposerai brièvement quelques aspects. 


\subsection{Lecture et construction du sens}

Le premier axe sur lequel il convient de travailler est celui de la construction du sens car c'est sur lui que va reposer la suite de l'opération. Les étudiants, lorsqu'ils font du thème, ne s'imaginent même pas avoir de problèmes de sens dans la mesure où ils travaillent sur un texte en langue maternelle; la conscience des problèmes devient plus aiguë avec la version où le problème du sens est alors lié et souvent réduit à la connaissance du vocabulaire (et accessoirement des fameuses expressions idiomatiques) ; je crois qu'il est bon dans un premier temps de rectifier cette vision étroite du sens en en montrant la complexité et en soulignant sa dépendance par rapport au processus de lecture.

On peut faire prendre conscience de la complexité de la construction du sens en en faisant apparaître les coordonnées: la situation d'énonciation, le contexte, etc., notions devenues classiques pour les spécialistes, mais dont il convient de faire saisir le rôle aux étudiants par des exercices d'application et d'observation. J'en donnerai deux exemples.

Le premier illustrera le rôle des repérages spatiaux (et de façon générale la deixis) dont beaucoup sont implicites pour le lecteur:

[la scène se situe à Londres pendant les bombardements; des officiers circulent dans la ville] They stood at the top of St James Street. Half way down Turtle's Club was burning briskly. From Piccadilly to the Palace the whole jumble of incongruous façades was caricatured by the blaze. (E.Waugh)

La traduction de «the Palace» par «le Palace» (rencontrée dans des copies de niveau CAPES) transforme Buckingham Palace en salle de spectacle ou en hôtel de luxe, et révèle une non-lecture des repères qui balisent très clairement les lieux de cette scène: St James Street et Piccadilly. Proposition de traduction:

Ils se trouvaient en haut de St James Street. À mi-hauteur, le Turtle's Club était la proie des flammes. De Piccadilly au Palais, tout ce mélange de façades hétéroclites se trouvait comme caricaturé par la lueur de l'incendie.

Le second exemple illustrera le rôle du contexte dans la construction du sens, et en particulier lorsque les anaphoriques sont impliqués; dans En attendant Godot, une des répliques de Vladimir a cette forme:

VLADIMIR: Un sur quatre. Des trois autres, deux n'en parlent pas du tout et le troisième dit qu'ils l'ont engueulé tous les deux. (Beckett: Godot: 15)

Il est difficile d'accéder au sens de cette phrase coupée de son contexte parce qu'elle comporte des chiffres et des anaphoriques. Tout au plus arrivera-t-on à déduire qu'il s'agit de personnes ayant eu un différend, mais qui sont-elles et quelle est la nature du différend? L'ensemble s'éclaire avec le contexte avant:

VLADIMIR: Comment se fait-il que des quatre évangélistes un seul présente les faits de cette façon? Ils étaient cependant là tous les quatre - enfin pas loin. Et un seul parle d'un larron sauvé. (Un temps) Voyons, Gogo, il faut me renvoyer la balle de temps en temps.

ESTRAgON: J'écoute. (Ibid) 
Dans la traduction anglaise, qu'il a faite lui-même, Beckett est plus explicite:

VLADIMIR: One of the four. Of the other three two don't mention any thieves at all and the third says that both of them abused him. (Beckett: 5)

Mais l'autre aspect sur lequel je voudrais attirer l'attention parce qu'il est plus souvent négligé ou pris comme allant de soi, c'est le degré d'implication du lecteur dans sa lecture sur le plan très élémentaire de la perception des signes. La lecture est d'abord une perception physique et elle demande une grande attention; le souligner et l'illustrer est une bonne façon d'impliquer l'étudiant dans l'acte et de le mettre face à ses responsabilités.

Il me semble donc bon de travailler dans un premier temps, à l'aide d'exercices, sur le fait que le traducteur ne part pas du sens, il part d'un texte constitué de formes signifiantes qu'il doit d'abord lire, au sens de percevoir, et dont il fait une interprétation afin de construire un sens qui sera celui qu'il attribue au texte. La traduction est donc d'abord une lecture. Or, cette opération n'est pas forcément assurée à tout coup; elle suppose que le traducteur est capable de lire correctement le texte sur au moins deux plans essentiels: sur le plan de la perception des formes et sur celui de l'interprétation des formes. S'il ne le fait pas, il risque de commettre un certain nombre d'erreurs.

La perception des formes par le lecteur n'est pas assurée s'il ne participe pas à la lecture avec toute son attention. C'est-à-dire qu'il doit être capable de percevoir non seulement ce qui est visible mais aussi invisible (ou implicite) ou anodin (je pense ici en particulier à la ponctuation). Je me contenterai ici d'illustrer la lecture du visible.

En principe, un bon traducteur doit s'efforcer de préserver le sens du texte qu'il traduit et pour cela il doit faire attention à ne rien ajouter et à ne rien retrancher qui risque d'altérer le sens de façon indue (il est bien évident qu'il y a des phénomènes d'ajout ou d'effacement qui sont nécessaires ou souhaitables, ils seront évoqués après). On peut observer ce phénomène à partir de productions d'étudiants ou de professionnels, encore une fois non pas par goût de la critique mais pour affûter l'esprit d'observation:

He was overgrown for his age, with a pale face that had bright blue eyes and a fall of chestnut hair on the forehead. (J. McGahern)

Il était trop grand pour son âge. Il avait un visage pâle, des yeux d'un bleu très vif et une masse de cheveux sur le front. (G.M. Sarotte)

Le traducteur n'a pas traduit chestnut, mais il est bien évident qu'il connaît ce mot, il s'agit donc d'un oubli (Proposition: «une masse de cheveux châtain »).

La conclusion que l'on peut en tirer pour les étudiants est que si des professionnels font ce type d'erreur c'est bien qu'eux-mêmes doivent faire attention. La pratique de ce type d'exercice a de plus un effet stimulant dans la mesure où il s'agit de relever les erreurs des autres.

On établira aussi la distinction entre ce qui ne doit pas se faire et ce qui peut se faire, et est même souhaitable. On distinguera l'omission (oubli ou suppression), qui est une faute, de l'effacement, qui est une opération de traduction (témoignant souvent du souci d'alléger le texte pour des raisons d'ordre stylistique), en voici un exemple:

The Ministry of Truth - Minitrue, in Newspeak - was startlingly different from any other object in sight. It was an enormous pyramidal structure of glittering white concrete, soar- 
ing up, terrace after terrace, 300 metres into the air. From where Winston stood it was just possible to read, picked out on its white face in elegant lettering, the three slogans of the Party [...]. (Orwell 1949; 1961: 7)

Le ministère de la Vérité - Miniver, en novlangue - frappait par sa différence avec les objets environnants. C'était une gigantesque construction pyramidale de béton d'un blanc éclatant. Elle étageait ses terrasses jusqu'à trois cents mètres de hauteur. De son poste d'observation, Winston pouvait encore déchiffrer sur la façade l'inscription artistique des trois slogans du Parti [...]. (Audiberti, 1950; 1972: 14-15)

On pourrait être tenté, à la première lecture, de critiquer cette traduction en faisant remarquer que "white» n'a pas été traduit dans le syntagme "white face», ce à quoi on peut répondre que cette soi-disant omission peut être interprétée de façon positive comme un souci d'éviter une répétition du terme «déjà » mentionné deux lignes plus haut en liaison avec le béton: "white concret». Mais, diront certains, si l'auteur a répété le terme, pourquoi ne pas le faire dans la traduction? Comme on le voit, un simple « détail » comme celui-là relance l'éternel débat de la traduction : selon l'original ou selon le discours d'arrivée?

Certains phénomènes d'étoffement, que je qualifierais d'idiomatiques, posent le problème de façon plus aiguë encore. On peut illustrer ce phénomène avec l'observation d'un court extrait de la traduction de The Spy Who Came in From the Cold. Rappelons, pour le contexte, que Leamas attend un agent qui doit franchir la frontière de Berlin-Est, celui-ci n'arrive pas et le membre des services qui accompagne Leamas lui fait remarquer ce retard. Leamas répond:

- Agents aren't airplanes. They don't have schedules. He's blown, he's on the run, he's frightened. Mundt's after him, now, at this moment. He's got only one chance. Let him choose his time. (John Le Carré 1963; 1978: 1-2)

- Les agents secrets ne sont pas des avions. Ils n'ont pas d'horaire fixe. Il est brûlé, il se sauve et il a peur. Mundt est à ses trousses, à l'heure qu'il est. Il a juste une petite chance de s'en sortir. Laissez-lui au moins choisir son heure. (Duhamel et Robillot, 1964; 1987: 8)

Si l'on compare les deux textes, on constate que le traducteur a pratiqué, d'instinct, trois étoffements: "petite», qui étoffe «chance»; «de s'en sortir» qui étoffe l'ensemble de la phrase; et «au moins» qui étoffe la dernière phrase. Il me semble qu'il y a peu de chances pour qu'un anglais à qui on donnerait ce texte à traduire pratique d'instinct ces étoffements - que certains critiqueront peut-être - et pourtant ils donnent à la traduction son caractère idiomatique; c'est une des raisons aussi pour lesquelles je pense (comme Peter Newmark, et la plupart des formateurs de traducteurs) que l'on ne doit traduire que vers sa langue maternelle.

\subsection{Reformulation et jugement}

Je traiterai brièvement de trois domaines: la déductibilité, le bagage cognitif, l'idiomatisme, où lecture/interprétation et reformulation s'interpénètrent et où se mêlent également un acte de jugement décidant de l'acceptabilité de la reformulation.

\section{La déductibilité}

Je pense que l'on peut aider les étudiants à résoudre leurs problèmes et à avancer dans la perception de la traduction en soulignant le fait qu 'une partie des solutions 
consiste à utiliser des paraphrases que l'on retrouve sur le plan intralinguistique. La différence de concentration est un paradigme permettant d'observer et d'utiliser ce phénomène. Il est un fait bien connu des traducteurs qu'un texte a tendance à s'allonger en traduction mais si l'on y regarde de plus prêt on peut observer en réalité des phénomènes symétriques: le développement et la réduction qui sont de l'ordre de la relation de la définition au terme. Par exemple:

- la réduction

I'd defied him in the most public way possible, I'd made a fool of him before not only his friends but also his enemies. (J. Braine)

Je l'avais défié de la manière la plus publique possible, je l'avais ridiculisé non seulement devant ses amis mais aussi devant ses ennemis.

- le développement

And from the bush there came the sound of little streams flowing, quickly, lightly, slipping between the smooth stones, gushing into ferny basins and out again;[...]. (K. Mansfield: 10)

Et de la brousse venait le son des ruisselets, qui coulaient vivement, légèrement, glissaient entre les pierres lisses, pénétraient, jaillissant, dans des vasques ombragées de fougères et en ressortaient. (M. Duproix: 209)

\section{Les référents culturels}

D'abord une définition: les référents culturels sont les signes renvoyant à des éléments ou des aspects caractéristiques d'une civilisation ou d'une culture. Il y a donc dans les référents culturels des noms propres et des noms communs (voir Ballard, 2001). Je voudrais montrer la manière dont les référents culturels nous renvoient à des problèmes fondamentaux de traduction qui tiennent à la fois à la lecture et à la reformulation, pour eux-mêmes, et de façon plus indirecte à des problèmes périphériques mais connexes. Je partirai de l'exemple suivant:

[une femme vient de prendre sa retraite]

'You could travel,' people had said and Letty had to agree with them, though it was not easy to imagine the kind of travelling she would do, alone on a package tour [...]

In the end her courage failed her and she got no further than studying the brochure, like Norman diving for buried treasure in Greece. Her attempts at 'travel' ended in a weekend to a distant cousin living in the West Country town where she had been born. (B. Pym)

Deux points me semblent révélateurs dans cet extrait: Norman et West Country.

Norman pose un problème à des Français tout d'abord parce qu'il aura tendance à être interprété comme signifiant «Normand» (de Normandie) d'où certaines traductions absurdes:

${ }^{\star}$ Elle ne fit rien de plus que de lire la brochure, comme ${ }^{\star}$ plongée normande pour un trésor enfoui en Grèce./comme ${ }^{\star}$ les normands plongeant en Grèce à la recherche de trésors enfouis. (étud. de CAPES)

*Elle n'était pas allé plus loin que la lecture de la brochure qui offrait des activités, à la manière des Normands qui plongeaient en quête de trésors cachés en Grèce. (étud. de CAPES)

Comme toujours, il me semble important de tirer du général à partir du particulier : ce cas nous renvoie à deux aspects de la traduction des noms propres: leur significa- 
tion, leur interprétation et leur traduisibilité. Cet exemple permet de bien illustrer ou de justifier le fait qu'il ne faut pas traduire les prénoms: on est tenté de le faire avec ceux qui ont une contrepartie dans l'autre langue, mais on s'aperçoit que certains n'en ont pas et que l'on aura alors des problèmes. Cette constatation est aussi l'occasion de percevoir qu'une culture est faite d'appellations spécifiques et aussi que le nom propre, bien que réputé être sans sens, signifie, mais avec des degrés de sollicitation divers: le nom propre renvoie ici à deux signes distincts: un prénom et un référent culturel dérivé d'un toponyme. Enfin, le problème nous renvoie aussi au fait qu'un texte à traduire est fait d'implicite, non immédiatement perceptible dans le contexte. Qui est Norman et que fait-il? Nous n'en savons rien.

En fin de compte, le courage lui avait manqué et elle n'avait pas dépassé la lecture de la brochure, un peu comme Norman rêvant de plongée sous-marine en Grèce, en quête de trésors engloutis. (Jury de CAPES)

Pour ce qui est de West Country voici tout une gamme d'erreurs constatée à partir de ce terme:

Her attempts at "travel" ended in a week-end to a distant cousin living in the West Country town where she had been born. (B. Pym)

1. Faux sens: le terme est interprété comme un nom de ville:

Sa tentative de «voyage» se termina par une visite, le temps d'un week-end, à un lointain cousin qui vivait à West Country, la ville où elle était née. (étudiant de CAPES) - qui vivait dans la ville de West Country $(i d$.

2. calque, opaque pour un public français:

Ses tentatives de voyage aboutirent à un week-end passé chez une cousine éloignée dans la ville du West country où elle était née. (id.)

3. sous-traduction ou inexactitude:

Sa tentative de voyage se solda par un week-end chez un lointain cousin vivant dans la ville de l'ouest où elle était née. (id)

Qui vivait à l'Ouest, dans la ville où elle était née. (id.)

Il y a sous-traduction parce que le lecteur français n'aura accès qu'à une localisation vague.

Alors que la traduction sera plus efficace en étant plus explicite:

Un week-end passé chez une cousine éloignée qui habitait la ville du Sud-Ouest de l'Angleterre où elle-même était née, mit un terme à ses velléités de «voyages».(jury de CAPES)

Cet exemple souligne les limites de la non traduction des noms propres référents culturels dont on dit toujours qu'il ne faut pas les traduire. À partir d'un cas comme celui-ci on peut remonter vers la gamme des traitements possibles et tenter d'éclairer ou de baliser les stratégies de traduction des référents culturels de façon ordonnée, construite, en soulignant bien la part de connaissance partagée sur laquelle reposent les choix définitifs. 


\section{L'idiomaticité de la traduction}

Après cet exemple révélateur des limites de la préservation de l'étrangeté du TD, si chère à certains théoriciens français, j'examinerai maintenant la question du calque si souvent incriminé comme vecteur de pollution linguistique par certains et comme nécessité ou violence souhaitable par d'autres. Le calque décrit une traduction littérale qui va à l'encontre des normes de la langue d'arrivée: il peut être perçu comme une erreur, une forme d'interférence, révélant une mauvaise gestion du bilinguisme sur lequel repose la traduction; il peut également être perçu comme une audace de traducteur, une manière d'obliger la langue d'arrivée à coller davantage au TD. Les avis diffèrent sur ce point et ces divergences donnent souvent lieu à des polémiques. Par exemple dans un article paru dans la revue Babel en 1974, Jean Maillot critique la traduction de Typhoon par Gide; il estime, entre autres, qu'il abuse du calque, et en particulier dans la traduction des expressions idiomatiques:

'For God's sake, Mr Jukes,' says he, 'do take away these rifles from the men. Somebody's sure to get hurt before long if you don't. Damme, if this ship isn't worse than Bedlam!' (Conrad 1903/1971: 225)

«Pour l'amour du Ciel, monsieur Jukes, enlevez-leur ces fusils. Vos hommes vont sûrement se blesser avec, si vous n'y veillez. Que le diable m'emporte si l'on ne se croirait pas à Bedlam. » (Gide 1918; 1985: 396)

Maillot estime que pour être compréhensible, il convient de dire: «on se croirait dans un asile de fous». Dix ans plus tard, Antoine Berman critique la position de Maillot et estime que Gide a eu raison de permettre au lecteur français d'accéder à cette expression anglaise. Les positions de Berman sont peut-être louables en tant que démarche d'ouverture à une autre culture mais, pratiquée de façon brutale et abrupte, cette politique ne mènera qu'à l'incompréhension, si vous donnez ce calque à lire à un public français il ne signifie rien (je l'ai testé auprès de plusieurs groupes d'étudiants entre autres). Il est évident que si l'on veut préserver cette expression, il faut l'accompagner d'une note, pour la clarifier et la rendre acceptable. Il est bon de se souvenir également que le calque pratiqué de façon systématique et non discriminée est un moteur comique que l'on trouve utilisé dans des bandes dessinée telles qu'Astérix ou dans des ouvrages comme Sky my Husband: Ciel mon mari! où le calque sert de repoussoir pédagogique. Faut-il donc, sous prétexte de calque visant à préserver la couleur de l'étranger, traduire de la façon suivante:

A free bus to the airport is a facility offered only by this hotel. (Longman)

Un bus gratuit pour l'aéroport est un service offert seulement par cet hôtel.

Une traduction (presque) littérale est déjà plus acceptable:

Un bus gratuit pour se rendre à l'aéroport est un service que seul cet hôtel offre.

On conserve le schéma général de l'énoncé, mais on applique des transformations minimales : étoffement de la préposition par verbe, transformation passif-actif dans la subordonnée relative. Mais l'on n'aboutit à une véritable traduction que par des aménagements plus poussés (thématisation de l'agent: «l'hôtel», posé comme sujet de la principale; le nom «transport» résulte d'une nominalisation du verbe «se rendre»; le «bus» devient un complément de moyen): 
Cet hôtel est le seul à offrir le transport gratuit par autobus jusqu'à l'aéroport.

Pour revenir à la traduction contestée par Berman, voici la traduction, très correcte (et compréhensible!) qui a été proposée du passage incriminé dans une traduction contemporaine :

«Pour l'amour du Ciel, monsieur Jukes, a-t-il continué, enlevez ces fusils à ces hommes avant que quelqu'un ne soit blessé. Que le diable m'emporte, ce navire est pire qu'une maison de fous!» (Ménard 1983; 1985: 186)

\section{Conclusions}

La traduction à l'université devrait se fixer des objectifs réalistes, tenant compte à la fois du monde réel, de la spécificité des études universitaires ainsi que des besoins et des capacités des individus au cœur de ce processus d'apprentissage : les étudiants. Le réalisme en matière d'objectifs consiste à tenir compte de la manière de traduire des professionnels ou mieux encore à tenir compte de la diversité des manières de traduire dans le monde en en examinant les raisons et les modalités. L'enseignement de la traduction n'a pas à être un endoctrinement, un dressage pour un moule d'action, ce serait contraire à la fonction de l'université, qui est d'être capable de prendre des distances avec un objet pour y réfléchir et en faciliter la perception. La traduction, parmi les matières enseignées à l'université est peut-être celle que l'étudiant sera le plus fréquemment et naturellement amené à réutiliser dans la vie réelle ultérieure au même titre que l'usage naturel de la langue; c'est donc une matière qui n'est pas à négliger mais elle mériterait d'être enseignée de façon différente plus élaborée, ordonnée autour d'un programme qui en souligne les modalités et favorise la réflexion.

\section{RÉFÉRENCES}

Ballard, M. (1994): La traduction: de l'anglais au français (1 $1^{\text {ère }}$ éd.) Paris, Nathan.

Ballard, M. (1992): Le commentaire de traduction anglaise, Paris, Nathan.

BALlard, M. (1998): «Les "mauvaises lectures" : étude du processus de compréhension » in Jean Delisle et Hannelore Lee-Jahnke (dir.), Enseignement de la traduction et traduction dans l'enseignement (Actes du colloque organisé par Maurice Pergnier à Créteil les 28, 29 et 30 avril 1997), Ottawa, Presses de l'Université, p. 27-47.

Ballard, M. (2001): Le Nom propre en traduction, Paris, Ophrys.

BALliu, C. (1999) : «La théorie est-elle nécessaire aux cours de traduction? », Linguistica Antverpiensa 33, p. 171-180.

Berman, A. (1985): «La Traduction et la lettre - ou l'auberge du lointain» in A. Berman et al., Les tours de Babel, Mauvezin, T.E.R., p. 31-179.

Chiflet, J.-L. (1985): Sky my Husband!/Ciel mon mari!, Paris, Éditions Hermé.

Delisle, J. (1980) L'analyse du discours comme méthode de traduction, réimpression, Ottawa, Presses de l'Université d'Ottawa.

Durieux, C. (1988) : Fondement didactique de la traduction technique, Paris, Didier.

Maillot, J. (1984): «Expressions idiomatiques en anglais et en français», Traduire, 119, p. 7-11. Van Der Meerschen, J.-M.: «Traduction française: problèmes de fidélité et de qualité », Lectures (Revue de l'Université de Bari) no spécial: 'Traduzione, tradizione', 4/5, agosto, p. 63-80. 